\title{
El lenguaje de los antropoides
}

\section{José Eugenio Ortega}

\author{
Universidad Autónoma de Madrid
}

\section{Desarrollo histórico}

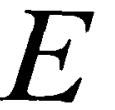

El aprendizaje del lenguaje por los animales siempre ha despertado interés entre los psicólogos y no es extraño que, a pesar de los fracasos, se hayan repetido los intentos. Witmer (1909) menciona el primer caso, un chimpancé llamado Peter, capaz de articular la palabra «mamá», aunque de forma imperfecta. Los Kellogg, un matrimonio de psicólogos, criaron juntos a su hijo Donald y a Gua, una chimpancé de corta edad. Los resultados fueron desalentadores: Gua no llegó a articular la palabra «papá» (Kellogg y Kellogg, 1933). Sin embargo, el trabajo de los Kellogg ha influido en gran parte de los intentos posteriores, principalmente por su insistencia en proporcionar al animal un ambiente humano que le facilitara el aprendizaje del lenguaje.

Los más cercanos al éxito fueron Keith y Catherine Hayes, que consiguieron enseñar a la chimpancé Viki, criada en su casa hasta los seis años, cuatro palabras: mama, papa, cup (copa) y up (arriba). La articulación de estos sonidos era imperfecta, aunque durante el entrenamiento, que duró varios años, se manipulaba la posición de los labios y la boca (Hayes y $\mathrm{Ha}$ yes, 1952).

\section{El proyecto Washoe}

El proyecto Washoe comenzó en 1966 y terminó en 1970. Durante los 51 meses que duró el experimento, la chimpancé Washoe adquirió 132 signos de Ameslan (Gardner y Gardner, 1969, 1975). El éxito de los Gardner se debió a la elección de un modo de comunicación no vocal. Los fracasos anteriores se habían interpretado como muestra de la incapacidad de los chimpancés de utilizar el lenguaje, exclusivamente humano; sin embargo, los Gardner argumientaban que se trataba más bien de una incapacidad articulatoria y conductual: 


\section{Estudios}

Un inconveniente más grave es el de que los sonidos del habla humana no son idóneos como medio de comunicación del chimpancé; pues su aparato fonador es muy distinto del que posee el hombre, $\mathrm{y}$, cosa más importante, el comportamiento vocal de tales animales difiere enormemente del que conocemos en éste: los chimpancés emiten, sí, muchos sonidos distintos, pero por lo general sus vocalizaciones aparecen en situaciones de gran excitación y tienden a ser propias sólo de éstas, en tanto que cuando no se les perturba suelen permanecer silenciosos. Así pues, es poco probable que sea posible adiestrar un chimpancé para que se valga de sus vocalizaciones con gran finura de empleo (Gardner y Gardner, 1976, pág. 26).

La influencia de este proyecto fue grande: en pocos años había varios equipos de investigadores trabajando desde distintas perspectivas y con diferentes metodologías. La mayoría utilizaba el lenguaje norteamericano de signos, ASL (Fouts, 1973; Patterson, 1978; Terrace et al., 1979). El grupo de Rumbaugh (Rumbaugh, Gill y von Glasersfeld, 1973; Savage-Rumbaugh, Rumbaugh y Boysen, 1980) utilizaba una consola de ordenador, con teclas para las distintas palabras o «lexigramas». Por último, Premack (1971) trabajaba con fichas de plástico. Estas diferencias metodológicas dificultan la comparación de los resultados de los distintos autores; por otra parte, ciertos medios de comunicación son más artificiales que otros. El más «natural» es el ASL. Yerkes ya había señalado que «quizás se les puede enseñar [a los chimpancés] a usar sus dedos, al igual que hacen los sordomudos, y de esta forma ayudarles a adquirir un "lenguaje de signos" sencillo, no vocal» (1925, pág. 179-180; citado en Seidenberg y Petitto, 1979). Esta «naturalidad» del ASL tendría por base la gran destreza manual de los primates, y permitiría explicar su adquisición en edades muy tempranas (Gardner y Gardner, 1975; Marchesi, 1981, proporciona una panorámica de los distintos lenguajes de signos y su adquisición).

\section{La elección de sujetos}

El sujeto favorito de los experimentadores ha sido el chimpancé, salvo dos excepciones: Patterson (1978), que enseñó el ASL a una gorila llamada Koko, y Furness (1916), que fracasó con el aprendizaje del lenguaje por un orangután. El chimpancé, el gorila y el orangután tienen una gran semejanza con el hombre y se les denomina, junto con el gibón, antropoides o antropomorfos. Aunque no hay un acuerdo completo entre los taxonomistas sobre la clasificación del orden primates, suele aceptarse la existencia de una superfamilia, Hominoidea, dividida en tres familias: Hylobatidae, Pongidae y Hominidae. El gibón pertenece a la primera familia, el chimpancé, el gorila y el orangután a la segunda, y el hombre a la tercera. Los póngidos son nuestros parientes más cercanos; no se conoce con exactitud - de momento- cuándo se produjo la divergencia entre el linaje humano y los de los antropoides. Los datos actuales indican que pudo ser hace unos 15 millones de años (Ayala, 1980).

Las capacidades craneanas medias de los póngidos son, aproximada- 
mente, $410 \mathrm{~cm}^{3}$ en el chimpancé, $510 \mathrm{~cm}^{3}$ en el gorila y $450 \mathrm{~cm}^{3}$ en el orangután, muy por debajo de los $1.500 \mathrm{~cm}^{3}$ del hombre actual (un estudio de los antropoides es el de Goustard, 1971). Además, tienen otras características que les convierten en sujetos idóneos para el tipo de estudios que se está considerando. Son animales sociables (en particular los chimpancés), aunque al alcanzar la madurez sexual se vuelven irascibles; disfrutan con las cosquillas y los juegos, y durante los dos primeros años dependen por completo de los adultos. No es extraño, por tanto, que la mayoría de los investigadores hayan seguido el procedimiento de los Kellogg de proporcionar un ambiente hogareño al animal joven (en Terrace et al., 1979, hay una descripción de la crianza de un chimpancé «lingüístico»). Debe señalarse que la interacción continua entre el antropoide y un reducido grupo de cuidadores plantea problemas a la hora de interpretar los resultados, como se verá más adelante.

\section{La polémica de los procedimientos}

Los diferentes procedimientos seguidos por los distintos investigadores, junto a la gran cantidad de tiempo y esfuerzo que se necesita dedicar a un animal para que aprenda un cierto vocabulario, han hecho surgir una viva polémica entre los distintos grupos. Cada uno argumenta que su metodología es correcta y que las restantes están plagadas de errores (dijo la sartén al cazo...). Así, los Gardner critican a Rumbaugh y a Premack porque, al utilizar pruebas de elección forzada, introducen indicios involuntarios del tipo Hans el Listo (1975, pág. 225). Savage-Rumbaugh et al. (1980) critican, a su vez, a todos los usuarios del ASL (los Gardner, Fouts, Patterson, Terrace y cols.) y a Premack; también introducen una crítica a su propio trabajo. Terrace et al. (1979) afirman que el trabajo de los Gardner (y los demás, por extensión) no es válido al carecer de un registro visual continuo en el que pudieran analizarse las situaciones y las conductas del mono y su maestro. Como puede observarse, en todos los trabajos de lenguaje de antropoides ha habido fallos en el control de variables; lo único discutible es hasta qué punto podían haberse evitado (sobre la imposibilidad de evitar las variables extrañas, vid. Sebeok y Umiker-Sebeok, 1979).

\section{Interpretación de los resultados}

Por lo dicho hasta aquí, no debe extrañar que existan tantas interpretaciones como investigadores. Simplificando un poco, encontramos las siguientes posturas:

1. Los antropoides poseen un verdadero lenguaje. Los partidarios de esta tesis interpretan las combinaciones de varios signos como equivalentes a las oraciones de los niños. Por ejemplo, los Gardner (1980, pág. 234) afirman que «si Washoe hubiera sido una niña de preescolar, entonces sus respuestas a las cuestiones - wh la situarían a un nivel relativamente avanzado de competencia lingüística, de acuerdo con las normas».

2. Los antropoides son capaces de aprender símbolos, pero no de 
combinarlos según una gramática. Las combinaciones de varios signos serían meras repeticiones, sin introducir cambios en el sentido. Los antropoides carecerían de la capacidad sintáctica necesaria para formar combinaciones con mayor valor informativo. Entre los partidarios de esta posición destacan Terrace, Petitto, Sanders y Bever (1979, pág. 901): «los antropoides pueden aprender muchos símbolos aislados (lo mismo que los perros, caballos y otras especies no humanas), pero no demuestran un dominio inequívoco de la organización convencional, semántica o sintáctica del lenguaje».

3. Los signos de los antropoides son una comunicación protolingüística. En la mayoría de los experimentos no se habría demostrado que los chimpancés estuvieran utilizando símbolos; la comunicación sería de carácter no lingüístico. Por ejemplo, Savage-Rumbaugh, Rumbaugh y Boysen afirman que «de hecho, la mayoría de los estudios de lenguaje en los antropoides no han sobrepasado realmente el nivel comunicativo básico del chimpancé» (1980, pág. 60). Esta posición es la que plantea menos problemas desde una perspectiva evolucionista, ya que niega que los chimpancés «humanos» tengan capacidades distintas de las de los chimpancés «salvajes». Naturalmente, la existencia de distintas capacidades sólo podría deberse a presiones selectivas que hubieran dejado de actuar en un momento determinado, conservándose la capacidad simbólica como un residuo que el chimpancé no necesitaría en su hábitat natural.

4. La utilización de signos por los antropoides es un artefacto experimental. Los partidarios de esta posición conceden gran importancia a los indicios involuntarios por parte de los experimentadores, así como a la interacción entre el mono y su entrenador; en lugar del aprendizaje, existiría un amaestramiento. Sebeok y Umiker-Sebeok (1979) presentan un análisis detallado de los posibles indicios involuntarios en el trabajo de los Gardner. Generalmente se ha malinterpretado esta posición y se ha reducido al efecto Hans. Aunque este último desempeña un papel importante, no es lo fundamental. Por tanto, los experimentos de doble ciego, aunque pueden controlar el efecto Hans, no suprimen toda la interacción. Como ejemplo, valga el siguiente:

Así pues, es posible que algunas de las diferencias que vemos en los tiempos medios de adquisición de los cuatro chimpancés puedan haberse debido a su comportamiento individual durante esta fase; y esto podría también explicar los malos resultados conseguidos por Cindy en la comprobación con doble desconocimiento. En efecto, durante la adquisición, a ésta le iba muy bien con la atención (tan de cerca) y los elogios que le dedicaba al experimentador; pero en la comprobación de la caja no había ni atención ni elogios: el observador sentado dentro de la jaula no entraba en interacción con ella, por temor de ver accidentalmente el objeto del interior de la caja, ni la elogiaba, puesto que no sabía si había respondido acertadamente o no; pues bien, durante estas comprobaciones, Cindy era el menos atento de los cuatro chimpancés, y su atención fue decreciendo progresivamente; al parecer, a medida que avanzaban. (Fouts, 1976, pāg. 67). 


\section{Estudios}

Aunque se haya comprobado el dominio de símbolos aislados, queda por demostrar que las combinaciones de signos no puedan explicarse de forma más sencilla. El chimpancé necesita la presencia de una persona durante la prueba, y las posibilidades de interacción son grandes. El único registro completo de diálogo en ASL entre un entrenador y un mono (Terrace et al., 1979 ) indica que los indicios involuntarios siempre estaban presentes.

\section{Implicaciones}

La continuidad mental entre el hombre y los otros animales ha sido siempre un tema polémico. El padre de la Psicología Comparada, Carlos Darwin, había escrito en 1871 (pág. 65) un capítulo destinado a «demostrar que no hay diferencia esencial en las facultades del hombre y mamíferos superiores». Para Darwin era crucial el principio de continuidad: el hombre habría evolucionado a partir de otros organismos, y la existencia de características exclusivamente humanas podría interpretarse como una negación de la evolución del hombre. El principal obstáculo era, naturalmente, el lenguaje; Darwin afirmaba que el hombre tendría una capacidad infinitamente superior para asociar sonidos con ideas, 'en función de su gran desarrollo cognoscitivo. Sin embargo, el lenguaje habría evolucionado a partir de la comunicación vocal de los primates.

La adquisición de vocabularios más o menos amplios por los simios vendría a reforzar el principio de continuidad, y plantearía un gran número de implicaciones en distintas disciplinas (desde la etología a la epistemología). Aunque no es momento para adoptar una posición final, no es posible dejar de preguntarse cuáles son las diferencias entre el hombre y los demás primates. Por lo que se conoce actualmente de los chimpancés, no parece que utilicen en su medio natural un sistema de comunicación tan complicado como el que adquieren en los experimentos de lenguaje de signos. Si es así, ¿cómo es posible que el chimpancé posea una capacidad simbólica en un medio en que no la necesita? Es un dogma científico desde los tiempos de Darwin que los caracteres evolucionan por presiones selectivas. ¿Cuáles han sido las presiones selectivas que han actuado sobre el chimpancé y el hombre para producir las distintas capacidades simbólicas? El estudio de los sistemas comunicativos naturales y de las capacidades de simbolización de los antropoides puede aportar bastantes datos al problema de la evolución humana, en la que el lenguaje ha debido desempeñar un papel crucial.

\section{Referencias}

AYALA, F. J.: Origen y evolución del hombre. Madrid: Alianza Universidad, 1980.

DARWIN, C. El origen del hombre. Madrid; EDAF, 1974 (original, 1871).

FOUTS, R. S. Acquisition and Testing of Gestural Signs in Four Young Chimpanzees. Science, 1973, vol. 180, 978-980 (versión cast. en Sánchez de Zavala, 1976).

FURNESS, W. H. Observations on the mentality of chimpanzees and orangutans. Proc. Amer. Phil. Soc., 1916, vol. 55, 281. 
GARDNER, B. T. y GARDNER, R. A. Evidence for sentence constituents in the early utterances of child and chimpanzee. Joumal of Experimental Psychology: General, 1975, 104, 244-267 (Versión cast. en este número).

GARDNER, R. A. y GARDNER, B. T. Teachin sign language to a chimpanzee. Science, 1969, 165, 664-672. (Versión cast. en Sánchez de Zavala, 1976).

GARDNER, R. A. y GARDNER, B. T. Comparative psychology and language acquisition. En Sebeok, T. A. y Umiker-Sebeok, J. (Eds.) Speaking of Apes, pág. 287-330. Nueva York: Plenum, 1980.

GOUSTARD, M. Los monos antropoides. Barcelona: Oikos-Tau, 1971.

HAYES, K. y HAYES, C. Imitation in a home-raised chimpanzee. Journal of Comparative and Psychological Psychology, 1952, 45, 450-459.

KELLOGG, W. N. y KELIOGG, L. A. The Ape and the Child: A Siudy of Environmental Influence on Early Behavior. Nueva York: McGraw-Hill, 1933.

MARCHESI, A. El lenguaje de signos. Estudios de Psicología, 1981, núms. 5 y 6.

PATTERSON, F. The gestures of a gorilla: Sign language acquisition in another pongid species. Brain and Language, 1978, 5, 72-97.

PREMACK, D. Some general characteristics of a method for teaching language to organisms that do not ordinarly acquire it. En L. E. Jarrard (ed.), Cognitive Processes of Nonbuman Primates. Nueva York: Academic, 1971, pág. 47-82 (Versión cast. en Sánchez de Zavala, 1976).

RUMBAUGH, D. M.; GILL, T. V. y VON GLASERSFELD, E. C. Reading and sentence completion by a chimpanzee (Pan). Science, 1973, vol. 182, pág. 731-3 (Versión cast. en Sánchez de Zavala, 1976).

SANCHEZ DE ZAVALA, V. (Comp.). Sobre el lenguaje de los antropoides. Madrid: Siglo XXI, 1976.

SAVAGE-RUMBAUGH, E. S.; RUMBAUGH, D. M. y BOYSEN, D. Do apes use language? American Scientist, 1980, 68, 49-61 (Versión cast. en este número).

SEBEOK, T. A. y UMIKER SEBEOK, J. Performing animals: Secrets of the trade Psychology Today, 1979, 13 (6), 78-91 (Versión cast. en este número).

SEBEOK. T. A. y UMIKER-SEBEOK, J. (Eds.) Speaking of apes. A critical anthology of two-way communication with man. Nueva York: Plenum, 1980.

SEIDENBERG, M. S. y PETITTO, L. A. Signing behavior in apes: A critical review. Cognition, 1979, vol. 2. 177-215.

TERRACE, H. S.; PETITTO, L. A.; SANDERS, R. J. y BEVER, T. G. Can an ape create a sentence? Science, 1979, 206, 891-902 (Versión cast. en este número).

WITMER, L. A monkey with a mind. The Psychological Clinic, 1909, vol. 3, núm. 7, 189-205.

YERKES, R. M. Almost Human. Nueva York: Century, 1925. 